\title{
In Vitro Activity of Colistin and Trimethoprim/Sulfamethoxazole Against Consortia of Multidrug Resistant Non-Fermenting Gram-Negative Bacilli Isolated from Lower Respiratory Tract
}

\author{
Emese Juhasz, ${ }^{1,}{ }^{*}$ Andrea Kovacs, ${ }^{1}$ Julia Pongracz, ${ }^{1}$ Miklos Ivan, ${ }^{1}$ and Katalin Kristof ${ }^{1}$ \\ ${ }^{1}$ Diagnostic Laboratory of Clinical Microbiology, Institute of Laboratory Medicine, Semmelweis University, Budapest, Hungary \\ "Corresponding author: Emese Juhasz, Semmelweis Univesity, Institute of Laboratory Medicine, Nagyvarad ter 4, 14. emelet, 1089, Budapest, Hungary. Tel: +36-14591500/62106, \\ E-mail: juhasz.emese@med.semmelweis-univ.hu
}

Received 2016 October 15; Revised 2017 March 11; Accepted 2017 March 18.

\begin{abstract}
Background: Multidrug resistant (MDR) Pseudomonas aeruginosa, Acinetobacter baumannii and Stenotrophomonas maltophilia have a leading role in nosocomial infections, including lower respiratory tract (LRT) infections. When polymicrobial infection by these three bacteria occurs, colistin against MDR P. aeruginosa and A. baumannii and trimethoprim/sulfamethoxazole (SXT) against S. maltophilia can be an optional antimicrobial strategy.

Objectives: The aim of this study was to investigate the potential synergic effect of colistin-plus-SXT against those MDR P. aeruginosa, A. baumannii and S. maltophilia isolates that were isolated at the same time, from the same LRT sample of patients.

Methods: Sixty connected isolates from 20 different patients were collected in a two-year study period. The checkerboard method and time-kill assays were used for synergy testing.

Results: All P. aeruginosa and A. baumannii strains were susceptible to colistin, whereas all S. maltophilia isolates were resistant to it. Fifteen percent of MDR A. baumannii strains and all S. maltophilia isolates were susceptible to SXT. By the checkerboard method, colistin-plus-SXT showed synergy in 50\%,35\% and 45\% of S. maltophilia, MDR P. aeruginosa and MDR A. baumannii strains, respectively. Antagonistic effect was not found. A time-kill assay was performed on strains which showed synergy by the checkerboard method: $70 \%, 57 \%$ and $56 \%$ of S. maltophilia, P. aeruginosa and A. baumannii strains showed the same results. Synergic activity of the combination was already detected after $6 \mathrm{~h}$ incubation in $86 \%$ of $S$. maltophilia isolates and $50 \%$ of $P$. aeruginosa strains. Regrowth of $A$. baumannii after 24 hour in the presence of colistin was prevented by the combination. The results gained by CB and TKA methods correlated in $61 \%$ of cases, but the $\Sigma$ FIC values did not correlate with the rate of log10 decrease in TKA. Colistin-plus-SXT combination had synergic effect on $35 \%$ of S. maltophilia, $20 \%$ of P. aeruginosa and $25 \%$ A. baumannii strains by both methods.

Conclusions: According to our in vitro results, colistin-plus-SXT combined therapy can be used efficiently in clinical practice as no antagonistic effect was detected. In certain cases colistin-plus-SXT has a synergic effect against MDR P. aeruginosa, A. baumannii and S. maltophilia.
\end{abstract}

Keywords: Colistin, Trimethoprim Sulfamethoxazole Drug Combination, Drug Synergism, Pseudomonas aeruginosa, Acinetobacter baumannii, Stenotrophomonas maltophilia

\section{Background}

Among the non-glucose-fermenting bacteria, Pseudomonas aeruginosa, Acinetobacter baumannii and Stenotrophomonas maltophilia have a leading role in nosocomial infections, especially in lower respiratory tract (LRT) infections in mechanically ventilated patients and in bacteraemia. While $S$. maltophilia has intrinsic resistance to many antibiotics, limiting treatment options to trimethoprim/sulfamethoxazole (SXT), fluoroquinolones and few other antibiotic agents, P. aeruginosa and A. baumannii often show a high level of acquired resistance in a hospital environment, often making colistin therapy necessary. The biofilm-forming ability of these bacteria makes antibiotic treatment even more challenging.
Polymicrobial colonization of the LRT is frequently observed in patients treated in intensive care units (ICU) or in patients suffering from chronic respiratory tract diseases with frequent hospital care. Polymicrobial infection can develop from previous polymicrobial colonization; however, it is difficult to decide whether the infection is really polymicrobial or caused by just one member of the bacterial consortia. Furthermore, differentiation between polymicrobial colonization and infection of the LRT in ventilated patients with serious underlying diseases is also difficult.

Stenotrophomonas maltophilia is often part of polymicrobial infections. In our centre $58 \%$ of S. maltophilia isolated from LRT specimens was cultured as co-pathogen or co-colonizer in 2013 - 2014. Pseudomonas aeruginosa was 
found to be the most frequent co-pathogen, but $A$. baumannii was also a significant co-habitant. Although coinfection/co-colonization by multidrug resistant (MDR) $P$. aeruginosa, A. baumannii and S. maltophilia in LRT is rare, we cannot treat it as a unique and therefore marginal problem. A rapid and efficacious antimicrobial therapy against this MDR bacterial consortium is essential.

In a meta-analysis it was demonstrated that colistin was efficacious and safe for treatment of patients with pulmonary infection caused by MDR P. aeruginosa or A. baumannii (1). However, considering the low penetration of colistin in the lung parenchyma after intravenous administration, there is a certain level of clinical reluctance to its use for treatment of respiratory tract infections. Inhalational use of colistin provides a high concentration in airways, and therefore represents a promising therapy approach (2). Trimethoprim/sulfamethoxazole is the firstline antimicrobial agent for S. maltophilia infections.

In cases of patients with MDR P. aeruginosa, MDR A. baumannii and S. maltophilia co-infection in LRT, colistin against MDR $P$. aeruginosa and A. baumannii plus SXT against $S$. maltophilia looks to be an optional or obligatory antimicrobial strategy. Colistin-plus-SXT is a combined monotherapy and not an unconventional combination therapy in such cases. The question is whether this 'combination' has synergy or antagonism on S. maltophilia, P. aeruginosa or A. baumannii.

In recent years combination antibiotic therapy has become an important option against MDR bacteria. Physicians should be supplied with in vitro synergy testing data, but most of the testing methods (checkekboard method, time-kill assay) are labour-intensive, therefore they are rarely performed in routine diagnostic laboratories. Furthermore, results gained by different techniques can be controversial and difficult to interpret. Especially nonfermenting bacteria demonstrate the methodical difficulties (3).

\section{Objectives}

Objective of this study was to determine the in vitro activity of the colistin-plus-SXT combination, using different synergy testing methods, against MDR P. aeruginosa, MDR A. baumannii and S. maltophilia strains isolated at the same time from the same LRT samples.

\section{Methods}

In a two-year study period (2013 - 2014) 392 consecutive non-duplicate S. maltophilia strains were isolated from LRT samples. In $58 \%$ of cases, other pathogens were isolated next to S. maltophilia. In 7\% of cases, both P. aeruginosa and A. baumannii were co-isolated and in $5 \%$ of cases $(n=20) P$. aeruginosa and $A$. baumannii fitted the criteria of multidrug resistance. This study included these 20 MDR $P$. aeruginosa, 20 MDRA. baumannii and 20 S. maltophilia isolates collected in the Diagnostic Laboratory of Clinical Microbiology, Institute of Laboratory Medicine, Semmelweis University (Budapest, Hungary). The bacterial 'triplets' were isolated at the same time from the same sample (tracheal aspirate or bronchoalveolar lavage sample) of different patients. Isolates were identified by the MALDI-TOF mass spectrometry technique (Bruker Daltonics, Germany). All strains were isolated from patients treated at ICUs.

Enterobacterial Repetitive Intergenic Consensus PCR (ERIC-PCR) was used for molecular typing of isolates, as described by Silbert et al. (4). Bacteria were suspended in $100 \mu \mathrm{L}$ of PCR-grade water and heated at $100^{\circ} \mathrm{C}$ for 15 minutes. After centrifugation at 12,000 rpm for 2 minutes, supernatant was removed. One $\mu \mathrm{L}$ of the supernatant was used as DNA for PCR. Primers of ERIC1 5'-ATGTAAGCTCCTGGGGATTCAC-3' and ERIC2 5'AAGTAAGTGACTGGGGTGAGCG-3' (Biocenter, Hungary) and REDTaq Ready Mix PCR reaction mix (Sigma-Aldrich, USA) were used for DNA amplification, in $50 \mu \mathrm{L}$ final PCR reaction volume. PCR conditions were the following: initial denaturation at $95^{\circ} \mathrm{C}$ for 2 minutes, 30 cycles at $90^{\circ} \mathrm{C}$ for 30 seconds, $52^{\circ} \mathrm{C}$ for 1 minute, $65^{\circ} \mathrm{C}$ for 8 minutes. Electrophoresis in $1.5 \%$ agarose gel stained with $0.01 \%$ GelRed (Biotium, USA) was performed. Isolates that differed by two or more bands were interpreted as unrelated.

Minimum inhibitory concentrations (MICs) were determined by the broth microdilution method in cationadjusted Mueller-Hinton II broth (Becton Dickinson, USA) (5). Escherichia coli ATCC 25922 and P. aeruginosa ATCC 27853 were used as quality control strains. Colistin (SigmaAldrich, USA) was tested in the range $1-512 \mathrm{mg} / \mathrm{L}$ in case of S. maltophilia strains and at $0.06-32 \mathrm{mg} / \mathrm{l}$ in case of $P$. aeruginosa, A. baumannii strains. The MIC values of SXT (Ratiopharm, Hungary) were tested at $0.5-256 \mathrm{mg} / \mathrm{L}$ and 2 $1024 \mathrm{mg} / \mathrm{L}$ in case of A. baumannii and P. aeruginosa strains, respectively, and at $0.06-32 \mathrm{mg} / \mathrm{L}$ in case of $S$. maltophilia isolates. To interpret MIC results, EUCAST species-specific breakpoints were applied, except for the colistin MIC of S. maltophilia, when the Pseudomonas sp.-specific breakpoint was used (6).

Antibiotic combination of colistin-plus-SXT was analysed initially by a checkerboard technique (CB). MuellerHinton II broth was used. Stenotrophomonas maltophilia isolates were tested in 7 doubling dilutions of colistin and 11 doubling dilutions of SXT, whereas $P$. aeruginosa and $A$. baumannii strains were tested in 7 doubling dilutions of SXT and 11 doubling dilutions of colistin. Microbroth plates 
were inoculated with bacteria to yield $5 \times 10^{5} \mathrm{CFU} / \mathrm{mL}$ in the $100 \mu \mathrm{L}$ final volume. Plates were incubated at $35^{\circ} \mathrm{C}$ for 18 - 22 hours. Fractional inhibitory concentration indices ( $\Sigma$ FIC) were calculated following the formula: FIC(A) $+\mathrm{FIC}(\mathrm{B})=\Sigma \mathrm{FIC}$, where $\mathrm{FIC}(\mathrm{A})=$ MIC of antibiotic agent $\mathrm{A}$ in combination/MIC of antibiotic agent $\mathrm{A}$ alone and $\mathrm{FIC}(\mathrm{B})=$ MIC of antibiotic agent $B$ in combination/MIC of antibiotic agent B alone (7). The $\Sigma$ FIC of two antibiotics tested defines the effects of antimicrobial agent combinations as antagonistic $(\Sigma$ FIC $>4)$, indifferent $(0.5<\Sigma$ FIC $\leq 4)$ or synergistic $(\mathrm{FICI} \leq 0.5)$.

When synergy was detected by $\mathrm{CB}$, a time-kill assay (TKA) was performed at 1xMIC following a previously published method (8). When MICs were above the therapeutic level, SXT was used at $8 \mathrm{mg} / \mathrm{L}$ and colistin at $4 \mathrm{mg} / \mathrm{L}$, which fits the peak serum levels of these agents (9). Twenty ml of SXT, colistin and SXT-plus-colistin containing MuellerHinton II broth were inoculated with bacteria to yield a density of $106 \mathrm{CFU} / \mathrm{mL}$ in the final volume. Tubes were incubated at $37^{\circ} \mathrm{C}$ with constant agitation. After 1, 2, 4, 6 and 24 hours incubation aliquots were removed, serially diluted in $0.9 \%$ sodium chloride solution and plated on sheep blood agar plates (BioMerieux, France). Colonyforming units (CFUs) were counted on agar plates after 24 hours incubation at $37^{\circ} \mathrm{C}$. The lower limit of detection by this method was $20 \mathrm{CFU} / \mathrm{mL}$. Synergy was defined as a $\geq 2$ log10 decrease in CFU/ml at $24 \mathrm{~h}$ for the antibiotic combination compared with its more active constituent (8).

\section{Results}

According to ERIC-PCR, isolates in the same species were from different genotypes. All $P$. aeruginosa and $A$. baumannii strains were susceptible to colistin, with $\mathrm{MIC}_{50}$ $1 \mathrm{mg} / \mathrm{L}$ and $\mathrm{MIC}_{90} 2 \mathrm{mg} / \mathrm{L}$. Fifteen percent of A. baumannii strains were susceptible to SXT, MIC $_{50} 32 \mathrm{mg} / \mathrm{L}$ and $\mathrm{MIC}_{90} 128 \mathrm{mg} / \mathrm{L}$ was found. Pseudomonas aeruginosa strains showed a high level of intrinsic resistance to SXT, with $\mathrm{MIC}_{50} 256 \mathrm{mg} / \mathrm{L}$ and $\mathrm{MIC}_{90} 512 \mathrm{mg} /$ L. All S. maltophilia strains were sensitive to SXT, with MIC $_{50} 0.25 \mathrm{mg} / \mathrm{L}$ and MIC ${ }_{90} 1$ $\mathrm{mg} / \mathrm{L}$, and resistant to colistin, with $\mathrm{MIC}_{50} 256 \mathrm{mg} / \mathrm{L}$ and $\mathrm{MIC}_{90}>512 \mathrm{mg} / \mathrm{L}$. Results of colistin-plus-SXT combination tests performed by CB method are summarized in Table 1. As summarized in Table 2 synergic and indifferent effects were found, but an antagonist effect was not.

Strains showing synergy by CB method were further examined by TKA. The tested S. maltophilia, P. aeruginosa and A. baumannii strains showed synergy in $70 \%, 57 \%$ and $56 \%$ of cases, respectively. The rates of log10 decrease after 6 and $24 \mathrm{~h}$ are summarized in Table 3 considering that for most combinations with colistin against Gram-negative species initial killing is usually dramatic, but is followed by significant regrowth. Synergic activity of the combination was already detected after 6 hours incubation in $86 \%$ of S. maltophilia isolates and $50 \%$ of $P$. aeruginosa strains. In the case of A. baumannii, synergy was detected just after 24 hours incubation. The results gained by CB and TKA methods correlated in $61 \%$ of cases, but the $\Sigma$ FIC values did not correlate with the rate of log10 decrease in TKA. The results of different in vitro synergy testing must be synthesized and carefully interpreted. Colistin-plus-SXT combination had synergistic effect on seven S. maltophilia (35\%), four P. aeruginosa (20\%) and five A. baumannii (25\%) strains by both methods.

\section{Discussion}

The potential synergic effect of colistin-plus-SXT against MDR $P$. aeruginosa, MDR A. baumannii and $S$. maltophilia isolates was investigated in this study. The isolates were connected as each one of the three species was isolated at the same time from the same LRT sample of patients. Colistin-plus-SXT therapy is an obligatory antimicrobial strategy in LRT co-infections caused by the discussed three bacteria.

Co-colonization of patients with carbapenem-resistant Enterobacteriaceae and A. baumannii or P. aeruginosa has been shown to be associated with increased antibiotic resistance and mortality (10). As potential interspecies interactions may enhance bacterial virulence and antibiotic resistance, co-colonization or co-infection of patients with the intrinsically carbapenem-resistant S. maltophilia and A. baumannii or P. aeruginosa might be associated with increased antibiotic resistance and mortality. This hypothesis was not considered in previous studies. In our study the patients' overall mortality in hospital was 50\%. This did not differ significantly from a previous study where all-cause mortality of $45 \%$ was found in 100 S. maltophilia infections, of which 62 cases were pneumonia (11). The high mortality underlines the need for a rapid and effective antimicrobial therapy.

The folate synthesis inhibitor SXT is the first-line antimicrobial drug for S. maltophilia infections. All S. maltophilia strains were sensitive to SXT in our study, which supports the current antimicrobial guidelines. Colistin was found to have weak in vitro activity against the studied S. maltophilia isolates: high level of colistin resistance $\left(\mathrm{MIC}_{50} 256 \mathrm{mg} / \mathrm{L}\right)$ was detected. This shows that colistin should not be used alone either in S. maltophilia infection or in S. maltophilia co-infection, but it can have synergic activity in combination, as reported in previous studies (12). The effect of colistin in antibiotic combination is based on its detergent-like property: it interacts with surface LPS 
Table 1. Summary of Results Gained by CB Method; Effect of Colistin-Plus-SXT Combination was Tested on 20 S. maltophilia, 20 MDR P. aeruginosa and 20 MDR A. baumannii Strains; Strains were Connected as Each One of the Three Species was Isolated at the Same Time from the Same LRT sample ${ }^{\mathrm{a}}$

\begin{tabular}{|c|c|c|c|c|c|c|c|c|c|c|c|c|c|c|c|}
\hline & \multicolumn{5}{|c|}{ Stenotrophomonas maltophilia } & \multicolumn{5}{|c|}{ Pseudomonas aeruginosa } & \multicolumn{5}{|c|}{ Acinetobacter baumannii } \\
\hline & AB1 & AB2 & $\mathrm{AB} 1+\mathrm{AB} 2$ & $\mathrm{AB} 2+\mathrm{AB} 1$ & $\sum \mathrm{FIC}$ & AB1 & $\mathrm{AB} 2$ & $\mathrm{AB} 1+\mathrm{AB} 2$ & $\mathrm{AB2}+\mathrm{AB} 1$ & $\sum \mathbf{F I C}$ & AB1 & AB2 & $\mathbf{A B 1}+\mathbf{A B} 2$ & $\mathrm{AB} 2+\mathrm{AB} 1$ & $\sum$ FIC \\
\hline 1 & 0.25 & 256 & 0.062 & 32 & 0.375 & 256 & 1 & 32 & 0.25 & 0.375 & 32 & 1 & 2 & 0.25 & 0.312 \\
\hline 2 & 0.062 & 256 & 0.062 & 4 & 1.015 & 128 & 1 & 8 & 1 & 1.06 & 8 & 2 & 1 & 0.25 & 0.25 \\
\hline 3 & 1 & 512 & 0.25 & 8 & 0.265 & 512 & 2 & 8 & 1 & 0.52 & 128 & 1 & 8 & 0.5 & 0.56 \\
\hline 4 & 1 & 8 & 0.5 & 2 & 0.75 & 128 & 2 & 8 & 1 & 0.56 & 64 & 2 & 4 & 0.25 & 0.187 \\
\hline 5 & 0.25 & 32 & 0.125 & 4 & 0.625 & 8 & 2 & 1 & 0.062 & 0.156 & 64 & 0.5 & 2 & 0.25 & 0.53 \\
\hline 6 & 0.25 & 256 & 0.062 & 8 & 0.281 & 512 & 2 & 8 & 1 & 0.52 & 32 & 2 & 4 & 0.5 & 0.375 \\
\hline 7 & 0.06 & 64 & 0.015 & 8 & 0.375 & 128 & 1 & 32 & 0.125 & 0.375 & 64 & 2 & 8 & 0.25 & 0.25 \\
\hline 8 & 0.5 & 512 & 0.062 & 16 & 0.151 & 16 & 2 & 0.5 & 1 & 0.53 & 32 & 2 & 16 & 0.25 & 0.625 \\
\hline 9 & 0.25 & 256 & 0.062 & 16 & 0.312 & 512 & 2 & 8 & 0.5 & 0.265 & 128 & 0.5 & 8 & 0.25 & 0.56 \\
\hline 10 & 0.5 & 32 & 0.062 & 2 & 0.187 & 512 & 2 & 8 & 1 & 0.52 & 32 & 1 & 2 & 0.5 & 0.56 \\
\hline 11 & 0.25 & 128 & 0.125 & 2 & 0.52 & 512 & 2 & 16 & 1 & 0.53 & 1 & 1 & 0.25 & 0.125 & 0.375 \\
\hline 12 & 0.125 & 256 & 0.125 & 16 & 1.06 & 512 & 1 & 32 & 0.5 & 0.56 & 1 & 1 & 0.25 & 0.125 & 0.375 \\
\hline 13 & 0.25 & 64 & 0.125 & 16 & 0.75 & 512 & 2 & 8 & 0.5 & 0.265 & 64 & 1 & 8 & 0.25 & 0.375 \\
\hline 14 & 0.5 & 512 & 0.062 & 16 & 0.25 & 8 & 1 & 1 & 0.25 & 0.375 & 128 & 0.5 & 4 & 0.25 & 0.53 \\
\hline 15 & 0.25 & 512 & 0.25 & 8 & 1.015 & 256 & 0.5 & 8 & 0.5 & 1.03 & 4 & 0.5 & 1 & 0.25 & 0.75 \\
\hline 16 & 0.25 & 512 & 0.25 & 8 & 1.015 & 512 & 1 & 16 & 0.5 & 0.53 & 2 & 0.5 & 1 & 0.25 & 1 \\
\hline 17 & 0.062 & 128 & 0.062 & 8 & 1.06 & 128 & 0.5 & 64 & 0.125 & 0.75 & 4 & 0.125 & 0.5 & 0.125 & 1.125 \\
\hline 18 & 0.25 & 256 & 0.25 & 8 & 1.03 & 1024 & 1 & 16 & 0.25 & 0.265 & 64 & 1 & 32 & 0.062 & 0.56 \\
\hline 19 & 0.5 & 128 & 0.125 & 8 & 0.375 & 16 & 0.5 & 4 & 0.25 & 0.75 & 64 & 0.5 & 8 & 0.125 & 0.375 \\
\hline 20 & 1 & 64 & 0.062 & 4 & 0.125 & 512 & 1 & 64 & 0.5 & 0.625 & 16 & 0.5 & 1 & 0.5 & 1.06 \\
\hline
\end{tabular}

${ }^{\mathrm{a}} \mathrm{AB1}$, MIC value of SXT; AB2, MIC value of colistin; $\mathrm{AB1}+\mathrm{AB2}$, MIC value of $\mathrm{SXT}$ in combination with colistin; $\mathrm{AB2}+\mathrm{AB1}$, MIC value of colistin in combination with SXT; $\Sigma$ FIC values in bold means synergism.

Table 2. Summary of Results Gained by CB Method; Colistin-plus-SXT Combination was Tested on 20 MDR P. aeruginosa, 20 MDRA. baumannii and 20 S. maltophilia Strains

\begin{tabular}{lcc}
\hline $\begin{array}{l}\text { Colistin + SXT Combination Tested by } \\
\text { CB Method }\end{array}$ & \multicolumn{2}{c}{ No. (\%) of Strains Showed } \\
\cline { 2 - 3 } & Synergy & Indifferent Effect \\
\hline S. maltophilia & $10(50)$ & $10(50)$ \\
\hline P. aeruginosa & $7(35)$ & $13(65)$ \\
\hline A. baumannii & $9(45)$ & $11(55)$ \\
\hline
\end{tabular}

and phospholipids, disturbing membrane permeability. Colistin exposure leads to increased permeability to large or hydrophobic compounds such as SXT (8). Synergic effect of colistin and SXT against S. maltophilia was found in $47 \%$ of isolates by Giamarellos-Bourboulis et al. (13). This is in concordance with our $\mathrm{CB}$ results (synergy in $50 \%$ of isolates). When $\mathrm{CB}$ and TKA results are evaluated together, the rate of synergic effect is only in $35 \%$.

In current medical practice SXT is not recommended for treatment of MDR Acinetobacter infections. In the majority of studies regarding MDR Acinetobacter spp., the non-susceptibility rate was $>70 \%$. In our study $85 \%$ of MDR A. baumannii strains were resistant to SXT. Only single case reports evaluated SXT for A. baumannii infections, mainly in combination therapy. Though they considered therapeutic success, clinical evidence has failed so far (14). Recent publication report that SXT combined with colistin might represent an effective therapy for severe carbapenem-resistant A. baumannii infections (15). In concordance with previously published data, colistin-plus-SXT was found to display a synergic effect against $A$. baumannii isolates: synergy was found in $45 \%$ by CB method, but in $25 \%$ when results gained by the two methods were synthesized. Similarly to the findings of Nepka et al. the regrowth of $A$. baumannii after 24 hours was prevented by colistinplus-SXT (15). In case of colistin-resistant A. baumannii strains colistin-plus-SXT combination demonstrated limited synergism (16).

Pseudomonas aeruginosa is a poor target for therapy with SXT (6). Strains showed high level of intrinsic resistance to SXT. The combination of colistin-plus-SXT was synergistic against $20 \%$ of $P$. aeruginosa. In contrast with our results, Vidaillac et al. found no activity of colistin-plusSXT against their tested colistin-susceptible $P$. aeruginosa strains (8).

Discrepancies between our results gained by $\mathrm{CB}$ and TKA indicate that different methods to assess synergic effects do not provide necessarily comparable results (17). Nevertheless, the probability of synergy is high in those cases when a synergic effect is proved by two different techniques. An important finding of our study is that colistin- 
Table 3. Summary of the Synergic Results Gained by TKA; Colistin-Plus-SXT Combination was Tested on Strains Which were Previously Tested by CB and $\Sigma$ FIC was $<0.5$

\begin{tabular}{|c|c|c|c|c|}
\hline \multirow{2}{*}{$\begin{array}{l}\text { Colistin + SXT Combination } \\
\text { Tested by TKA }\end{array}$} & \multicolumn{3}{|c|}{ No. (\%) of strains showed synergy by TKA } & \multirow{2}{*}{$\begin{array}{l}\text { Previously Determined } \\
\qquad \text { FIC Values }\end{array}$} \\
\hline & Synergy by TKA & Difference in log10 after $6 h$ & Difference in log10 after $24 \mathrm{~h}$ & \\
\hline \multirow[t]{6}{*}{ S. maltophilia } & $7(70)$ & 5.9 & 2.4 & Sm\#1 $\Sigma F I C=0.375$ \\
\hline & & 7.7 & 3.8 & $\mathrm{Sm} \# 3 \Sigma \mathrm{FIC}=0.265$ \\
\hline & & 7.5 & 3.9 & Sm\#6 $\Sigma$ FIC $=0.281$ \\
\hline & & 2.7 & 2.9 & Sm\#9 $\Sigma F I C=0.312$ \\
\hline & & 6.3 & 3.1 & Sm\#10 $\Sigma$ FIC $=0.187$ \\
\hline & & 6 & 6 & Sm\#20 $\Sigma$ FIC $=0.125$ \\
\hline \multirow[t]{4}{*}{ P. aeruginosa } & $4(57)$ & 7 & 4.2 & Pa $\# 1 \Sigma F I C=0.375$ \\
\hline & & 3.4 & 2.3 & 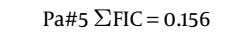 \\
\hline & & 6.7 & 4.1 & Pa\#14 $\Sigma F I C=0.375$ \\
\hline & & 7.2 & 3.7 & Pa\#18 $\Sigma$ FIC $=0.266$ \\
\hline \multirow[t]{5}{*}{ A. baumannii } & $5(56)$ & 3.1 & 7.3 & $\mathrm{Ab} \# 2 \Sigma \mathrm{FIC}=0.25$ \\
\hline & & 3.3 & 5.3 & $\mathrm{Ab \# 4} \Sigma \mathrm{FIC}=0.094$ \\
\hline & & 3.1 & 7.3 & $\mathrm{Ab} \# 6 \Sigma \mathrm{FIC}=0.375$ \\
\hline & & 2.7 & 4.8 & $\mathrm{Ab} \# 11 \Sigma \mathrm{FIC}=0.375$ \\
\hline & & 3.6 & 5.5 & $\mathrm{Ab} \# 12 \Sigma \mathrm{FIC}=0.375$ \\
\hline
\end{tabular}

plus-SXT combination can be used efficiently as no antagonistic effect was detected. Furthermore, synergism can be observed in $20 \%$ - $35 \%$ of isolates. Regrowth of A. baumannii after 24 hour in the presence of colistin can be prevented by colistin-plus-SXT combination. Of note, previous studies tested each species separately, whereas in our study MDR bacteria were investigated in their complex 'triplet' as they were isolated from a LRT sample. Two 'triplets' out of 20 showed synergy verified by both methods. In these cases patients had obvious benefit from combined colistinplus-SXT therapy.

The potential interspecies interaction between these bacteria has to be highlighted. Dominantly in cystic fibrosis several studies focused on interaction of $P$. aeruginosa with other bacterial species, but only a few have been published on the interaction between P. aeruginosa and S. maltophilia. It was found that S. maltophilia increases the risk of resistance of $P$. aeruginosa to polymyxin; beta-lactamase leaking from S. maltophilia enhances the growth of P. aeruginosa in the presence of beta-lactam antibiotic agents; $S$. maltophilia might confer a selective fitness advantage to $P$. aeruginosa and increase the virulence of $P$. aeruginosa (18). The interaction of A. baumannii and S. maltophilia is not discussed in the literature, except for their ability to increase each other's biofilm production (19). It was reported that a Burkholderia cenocepacia subpopulation highly resistant to polymyxin B can protect a sensitive $P$. aeruginosa from polymyxin B in broth co-culture (20). Similarly, it can be hypothesized that S. maltophilia highly resistant to colistin can protect a sensitive $P$. aeruginosa or A. baumannii from colistin in broth co-culture. Co-culturing of these bacteria in sessile form - like they growth together in LRT biofilms - can be suitable to detect this presumed interaction. Further investigations are needed to elucidate this hypothesis.

Further in vitro pharmacokinetic/pharmacodynamic experiments and animal studies are required to evaluate the combination of colistin with SXT against MDR Gramnegative pathogens. Evaluation of the clinical significance of our observation has to be performed also. The doseresponse relationship of the colistin-plus-SXT combination must be clarified.

In conclusion, according to our in vitro results we can state that colistin-plus-SXT combined therapy can be used efficiently in clinical practice as no antagonistic effect was detected. In certain cases colistin-plus-SXT has a synergic effect against MDR P. aeruginosa, A. baumannii and S. maltophilia.

\section{Footnotes}

Authors' Contribution: Study concept and design: Emese Juhasz, Katalin Kristof; aquisition of data: Emese 
Juhasz, Andrea Kovacs; analysis and interpretation of data: Emese Juhasz, Andrea Kovacs; drafting of the manuscript: Emese Juhasz; critical revision of the manuscript for important intellectual content: Emese Juhasz, Katalin Kristof; statistical analysis: Emese Juhasz, Andrea Kovacs, Miklos Ivan; administrative, technical and material support: Emese Juhasz, Andrea Kovacs, Miklos Ivan, Julia Pongracz; study supervision: Emese Juhasz, Katalin Kristof.

Funding/Support: The study was not funded.

Financial Disclosure: Authors declare that no competing financial interests exist.

\section{References}

1. Zhang H, Zhang Q. Clinical efficacy and safety of colistin treatment in patients with pulmonary infection caused by Pseudomonas aeruginosa or Acinetobacter baumannii: a meta-analysis. Arch Med Sci. 2015;11(1):34-42. doi:10.5114/aoms.2015.48158. [PubMed: 25861288].

2. Demirdal T, Sari US, Nemli SA. Is inhaled colistin beneficial in ventilator associated pneumonia or nosocomial pneumonia caused by Acinetobacter baumannii?. Ann Clin Microbiol Antimicrob. 2016;15:11. doi: 10.1186/s12941-016-0123-7. [PubMed: 26911714].

3. van Belkum A, Halimi D, Bonetti EJ, Renzi G, Cherkaoui A, Sauvonnet $\mathrm{V}$, et al. Meropenem/colistin synergy testing for multidrug-resistant Acinetobacter baumannii strains by a two-dimensional gradient technique applicable in routine microbiology.JAntimicrob Chemother. 2015;70(1):167-72. doi:10.1093/jac/dku342. [PubMed: 25239465].

4. Silbert S, Pfaller MA, Hollis RJ, Barth AL, Sader HS. Evaluation of three molecular typing techniques for nonfermentative Gramnegative bacilli. Infect Control Hosp Epidemiol. 2004;25(10):847-51. doi: 10.1086/502307. [PubMed: 15518027].

5. Institute CaLS . Performance Standards for Antimicrobial Susceptibility testing. CLSI approved Standard. Wayne: CLSI; 2014.

6. EUCAST . Breakpoints 2014. Available from: http://www.eucast.org/ clinical_breakpoints/.

7. Garcia L. Clinical Microbiology Procedures Handbook. Washington DC: ASM Press; 2010

8. Vidaillac C, Benichou L, Duval RE. In vitro synergy of colistin combinations against colistin-resistant Acinetobacter baumannii, Pseudomonas aeruginosa, and Klebsiella pneumoniae isolates. Antimicrob Agents Chemother. 2012;56(9):4856-61. doi: 10.1128/AAC.05996-11. [PubMed: 22751540].

9. Kadar B, Kocsis B, Toth A, Damjanova I, Szasz M, Kristof K, et al. Synergistic antibiotic combinations for colistin-resistant Klebsiella pneumoniae. Acta Microbiol Immunol Hung. 2013;60(2):201-9. doi: 10.1556/AMicr.60.2013.2.10. [PubMed: 23827751].
10. Marchaim D, Perez F, Lee J, Bheemreddy S, Hujer AM, Rudin S, et al. "Swimming in resistance": Co-colonization with carbapenemresistant Enterobacteriaceae and Acinetobacter baumannii or Pseudomonas aeruginosa. Am J Infect Control. 2012;40(9):830-5. doi: 10.1016/j.ajic.2011.10.013. [PubMed: 22325727].

11. Juhasz E, Krizsan G, Lengyel G, Grosz G, Pongracz J, Kristof K. Infection and colonization by Stenotrophomonas maltophilia: antimicrobial susceptibility and clinical background of strains isolated at a tertiary care centre in Hungary. Ann Clin Microbiol Antimicrob. 2014;13:333. doi: 10.1186/s12941-014-0058-9. [PubMed: 25551459].

12. Abbott IJ, Slavin MA, Turnidge JD, Thursky KA, Worth LJ Stenotrophomonas maltophilia: emerging disease patterns and challenges for treatment. Expert Rev Anti Infect Ther. 2011;9(4):471-88. doi: 10.1586/eri.11.24. [PubMed: 21504403].

13. Giamarellos-Bourboulis EJ, Karnesis L, Giamarellou H. Synergy of colistin with rifampin and trimethoprim/sulfamethoxazole on multidrug-resistant Stenotrophomonas maltophilia. Diagn Microbiol Infect Dis. 2002;44(3):259-63. [PubMed:12493173].

14. Falagas ME, Vardakas KZ, Roussos NS. Trimethoprim/sulfamethoxazole for Acinetobacter spp.: A review of current microbiological and clinical evidence. Int J Antimicrob Agents. 2015;46(3):231-41. doi: 10.1016/j.ijantimicag.2015.04.002. [PubMed: 26070662].

15. Nepka M, Perivolioti E, Kraniotaki E, Politi L, Tsakris A, Pournaras S. In Vitro Bactericidal Activity of Trimethoprim-Sulfamethoxazole Alone and in Combination with Colistin against CarbapenemResistant Acinetobacter baumannii Clinical Isolates. Antimicrob Agents Chemother. 2016;60(11):6903-6. doi: 10.1128/AAC.01082-16. [PubMed: 27550356].

16. Bae S, Kim MC, Park SJ, Kim HS, Sung H, Kim MN, et al. In Vitro Synergistic Activity of Antimicrobial Agents in Combination against Clinical Isolates of Colistin-Resistant Acinetobacter baumannii. Antimicrob Agents Chemother. 2016;60(11):6774-9. doi: 10.1128/AAC.00839-16. [PubMed: 27600048].

17. Doern CD. When does 2 plus 2 equal 5? A review of antimicrobial synergy testing. J Clin Microbiol. 2014;52(12):4124-8. doi:10.1128/JCM.0112114. [PubMed: 24920779].

18. Pompilio A, Crocetta V, De Nicola S, Verginelli F, Fiscarelli E, Di Bonaventura G. Cooperative pathogenicity in cystic fibrosis: Stenotrophomonas maltophilia modulates Pseudomonas aeruginosa virulence in mixed biofilm. Front Microbiol. 2015;6:951. doi 10.3389/fmicb.2015.00951. [PubMed: 26441885].

19. Varposhti M, Entezari F, Feizabadi MM. Synergistic interactions in mixed-species biofilms of pathogenic bacteria from the respiratory tract. Rev Soc Bras Med Trop. 2014;47(5):649-52. [PubMed: 25467269].

20. El-Halfawy OM, Valvano MA. Chemical communication of antibiotic resistance by a highly resistant subpopulation of bacterial cells. PLoS One. 2013;8(7):e68874. doi: 10.1371/journal.pone.0068874. [PubMed 23844246]. 